\title{
Feedback control of parametrized PDEs via model order reduction and dynamic programming principle
}

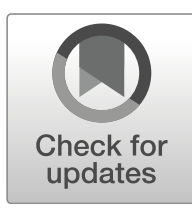

\author{
Alessandro Alla ${ }^{1}$ (D) Bernard Haasdonk ${ }^{2} \cdot$ Andreas Schmidt $^{2}$ \\ Received: 26 September 2018 / Accepted: 3 January 2020 / \\ Published online: 13 February 2020 \\ (C) Springer Science+Business Media, LLC, part of Springer Nature 2020
}

\begin{abstract}
In this paper, we investigate infinite horizon optimal control problems for parametrized partial differential equations. We are interested in feedback control via dynamic programming equations which is well-known to suffer from the curse of dimensionality. Thus, we apply parametric model order reduction techniques to construct low-dimensional subspaces with suitable information on the control problem, where the dynamic programming equations can be approximated. To guarantee a low number of basis functions, we combine recent basis generation methods and parameter partitioning techniques. Furthermore, we present a novel technique to construct non-uniform grids in the reduced domain, which is based on statistical information. Finally, we discuss numerical examples to illustrate the effectiveness of the proposed methods for PDEs in two space dimensions.
\end{abstract}

Keywords Dynamic programming principle - Semi-Lagrangian schemes · Hamilton-Jacobi-Bellman equations · Optimal control · Model reduction · Reduced basis method

Mathematics Subject Classification (2010) 49L20 • 49J20 • 65N99 · 78M34

\section{Introduction}

Optimal control problems are challenging tasks with a huge impact in real-life applications. The overall goal of control is to modify the behavior of dynamical systems

Communicated by: Anthony Nouy

This article belongs to the Topical Collection: Model Reduction of Parametrized Systems Guest Editors: Anthony Nouy, Peter Benner, Mario Ohlberger, Gianluigi Rozza, Karsten Urban and Karen Willcox

Alessandro Alla

alla@mat.puc-rio.br

Extended author information available on the last page of the article. 
through an external source, referred to as the control, chosen such that we are able to steer the solution trajectory to desired configurations or to achieve certain stability and performance goals. The field of open-loop PDE constrained optimal control is a quite "classical" area with plenty of progress both in theoretical and algorithmical aspects. We refer to the monographs [24] and [38] as some general references. Based on a mathematical model of a system at hand, an optimal control signal can be computed and then applied to the real system. However, hereby the optimal control signal is precomputed and not able to react on instantaneous changes of the system or environment. This problem is solved by closed-loop control, i.e., feedback control strategies. Thus, from an application perspective, we are in particular interested in optimal controls which stabilize the system even under perturbations. This is a crucial point due to errors in the measurements and the inherent non-exactness of mathematical models of real-life applications.

In this work, we aim at the control of "parametrized" problems in feedback form, where the parameters can describe, e.g., different material parameters, geometry modifications, or model uncertainties. Usually, one is interested in solving control problems for many different parameters, e.g., in parameter studies, Monte-Carlo simulations, or real-time parameter updates. This is often referred to as "multi-query" scenarios.

A general framework for feedback control has been introduced by Bellman in [10] in the 1950s via the Dynamic Programming Principle (DPP) which provides an efficient tool for the computation of the so-called value function, which is an important ingredient for feedback control. This approach is rather general and includes different optimal control problems such as, e.g., the minimum time problem, and the discounted infinite horizon control problem. The knowledge of the value function provides plenty of information on the control problem. Among others, we are able to compute an optimal policy for different initial conditions and/or under perturbation of the dynamical system. This is a huge advantage with respect to the more popular approach based on an open-loop strategy.

However, the method requires the solution of a non-linear partial differential equation (PDE), e.g., the Hamilton-Jacobi-Bellman (HJB) equation, whose dimension corresponds to the dimension of the underlying control problem (see, e.g., [8]). Due to the non-linearity of the HJB equation, it is usually not possible to derive analytical solutions. Thus, it is crucial to investigate numerical algorithms to build approximations of the value functions. Unfortunately, classical numerical methods suffer from the so-called curse of dimensionality. Although theoretical results hold true in any dimension, the computational approximation constitutes the bottleneck of this approach.

Many numerical methods deal with the approximation of the solution to the HJB equation, such as finite volume, finite element, and finite difference methods. We refer to the monograph [18] and the references therein for an extensive presentation of suitable numerical methods. Recently, new techniques such as radial basis functions (e.g., [26, 35]), sparse grid methods (e.g., [19]), and a tree structure algorithm ([3]) have been investigated for HJB equations. 
In the current work, we will deal with semi-Lagrangian (SL) schemes which provide stable approximations of the value functions even for coarse discretizations. We make use of accelerated iterative schemes based on the fixed point iteration introduced in [2] where a smart coupling between a value iteration (see, e.g., [8, Appendix A]) scheme and policy iteration (see, e.g., [13]) can drastically decrease the computational time to determine the numerical approximation.

Due to memory limitations, we are typically able to approximate HJB equations only up to a relatively low dimension of say $4-5$ dimensions, with a SL scheme, which is a big restriction in applications since the spatial dimension of the HJB equation is the same as the state dimension of the dynamical system. For instance, semi-discretizations of PDEs lead to a very large number of ordinary differential equations (ODEs) which makes this approach not feasible since the dimension can easily have $n \gg 10,000$ states or more, which would lead to memory requirements of the order of $\mathcal{O}\left(1 / h^{n}\right)$, where $h$ is a discretization parameter, for example the grid-width of a uniform grid.

One way to overcome these difficulties for PDE-related applications is to apply model order reduction (MOR) to the dynamical systems in the first place. MOR methods (see, e.g., [11] and the references therein) are (typically) projection-based methods that have been successfully applied to different problems such as optimization and many-query problems to reduce the number of degrees of freedom of the problem and to obtain surrogate models that represent the full-dimensional and expensive model accurately. Although a detailed description of model reduction techniques goes beyond the scope of this work, we want to mention proper orthogonal decomposition (POD, see [21]) and balanced truncation (BT, see [6]) as two of the most popular techniques for the reduction of dynamical systems. POD is a rather general method, which is based on a Galerkin projection of the dynamical system onto a space whose basis functions are built upon snapshots of the system state. Instead, BT is based on a Petrov-Galerkin projection, where the basis functions are obtained by solving two Lyapunov equations involving the system matrices. By construction, POD is particularly suitable to approximate trajectories, and BT for the approximation of the system's input/output behavior. The reduced basis (RB) approach deals with parametric problems based on greedy algorithms (see, e.g., [22, 32]). In this work, we mainly focus on the latter approach.

The POD method has been coupled with the HJB equations in the pioneering paper studies $[7,30,31]$ to compute feedback controls for high-dimensional problems including both linear and non-linear problems. Other features of the method have been investigated such as a priori error estimates [4] and the chattering of the feedback control [2]. Other model reduction methods have been coupled with the HJB approach, such as BT [27] and more recently a comparison of reduced order modeling (ROM) techniques has been conducted in [5]. For the sake of completeness, we would like to mention that BT has been applied to the Linear Quadratic Gaussian (LQG) problems which involves the solution of two Algebraic Riccati Equations to obtain a robust control for linear models (see, e.g., [12, 14]). Other approaches for the control of PDEs via the DPP deal with sparse grids for linear problems (see, 
e.g., [19]) and spectral elements for unconstrained controls (see, e.g., [28]). In contrast, the SL method is rather general and includes control constraints and non-linear dynamical systems. As already mentioned, the focus of this work is the computation of feedback control functions for parametrized PDEs via the HJB equation coupled with MOR techniques. To the best of our knowledge, this approach has not been investigated yet. For completeness, we want to mention some references on applying MOR in classical open-loop optimal control, in particular POD-based approaches have been presented in, e.g., [33, 39], and parametric settings in, e.g., [15, 29]. In contrast to these, in the current paper, we aim at closed-loop feedback control. In order to tackle this problem, we propose a complete workflow for the coupling of non-linear feedback control via HJB equations and MOR. While several single methodological ingredients of our scheme may be well known, the overall combination of those techniques is to be understood as the main contribution of the current paper. We also remark that MOR is extremely necessary for the feasibility of the problem. This means that in contrast to other MOR methods, the purpose of the approach is not acceleration of an existing scheme, but enabling feasability of HJB-based feedbackcontrol for parametric PDEs.

Starting from a general problem formulation, we first make use of recent ideas $[5,36]$ to project the control problem onto low-dimensional subspaces. Faced with parameter-dependent problems and with the requirement of very low-dimensional subspaces, we employ adaptive parameter partitioning techniques to reach spaces of dimension, say, maximum 5. For the actual numerical approximation, we employ the SL scheme for which a grid in the reduced space is required. To this end, we introduce a novel idea based on statistical assumptions on the highdimensional system that enables data-driven approximation of the relevant part of the reduced space which is then covered by a grid. Finally, an efficient offlineonline splitting is introduced to enhance and accelerate the overall procedure. In particular, we take advantage of the so-called value iteration (VI) scheme to precompute the value function in the barycenter of each parameter subregion in the offline phase and then switch, in the online phase, to the policy iteration (PI) method using the precomputed information on the value function as initial guess. This turns out to be a very efficient method as discussed in the numerical tests.

To summarize, the novelties in this paper are the following: (i) We consider a novel problem type by the presence of parameters for HJB-based non-linear feedback control problems. Further, several methodological ingredients emerge, namely (ii) the use of basis functions which do not depend on any control input, (iii) an automatic way to generate the domain for the reduced HJB equation, and (iv) an efficient offline-online scheme.

To set the paper into perspective, we recall the DPP approach and its numerical approximation in Section 2. Section 3 focuses on MOR for the HJB equation and all the building blocks for our approach. Finally, numerical experiments are presented in Section 4 with focus on the control of two-dimensional unsteady PDEs. Conclusions and future directions are discussed in Section 5. 


\section{Numerical methods for dynamic programming equations}

In this section, we recall the basic results for the numerical approximation of the HJB equations, additional details can be found in, e.g., [8] and [18]. Consider a continuous-time, parametric optimal control problem of the form:

$$
\begin{array}{r}
\min _{u \in \mathcal{U}} J_{x}(u ; \mu), \quad \text { with } \quad J_{x}(u ; \mu):=\int_{0}^{\infty} g(y(s), u(s) ; \mu) e^{-\lambda s} d s \\
\text { subject to } \dot{y}(t ; \mu)=f(y(t), u(t) ; \mu), \quad y(0 ; \mu)=x,
\end{array}
$$

with system state $y(t ; \mu)$ in $\mathbb{R}^{n}$ for $t \geq 0$, an initial state $x \in \mathbb{R}^{n}$ and a control signal $u \in \mathcal{U}$ with

$$
\mathcal{U} \equiv\{u:[0, \infty) \rightarrow U, \text { measurable }\},
$$

where $U$ is a compact subset of $\mathbb{R}^{m}$ of admissible control values and $\lambda>0$ is the discount factor. We consider the dynamics and the cost functional to be parametrized by a parameter vector $\mu \in \mathcal{P} \subset \mathbb{R}^{q}$, where $\mathcal{P}$ is a bounded set of admissible parameters. The following statements and definitions are to be understood to hold for any $\mu \in \mathcal{P}$. We assume that the running costs $g(\cdot, \cdot ; \mu)$ and the dynamics $f(\cdot, \cdot ; \mu)$ are Lipschitzcontinuous functions in the first two variables. Under rather general assumptions, the existence and uniqueness of solutions to the optimal control systems are guaranteed (see, e.g., [8]). A crucial tool in feedback control is the value function, which provides the minimum value of the cost functional at each point in the state space $x \in \mathbb{R}^{n}$. For parametric problems we define it as

$$
v: \mathbb{R}^{n} \times \mathcal{P} \rightarrow \mathbb{R}, \quad v(x ; \mu):=\inf _{u \in \mathcal{U}} J_{x}(u ; \mu),
$$

and its characterization through the DPP for $\tau>0$

$$
v(x ; \mu)=\inf _{u \in \mathcal{U}}\left\{\int_{0}^{\tau} g\left(y_{x}(t, u ; \mu), u(t) ; \mu\right) \mathrm{e}^{-\lambda t} \mathrm{~d} t+v\left(y_{x}(\tau, u ; \mu), u ; \mu\right) \mathrm{e}^{-\lambda \tau}\right\},
$$

where we denote by $y_{x}(t, u ; \mu)$ the state of the system at time $t$ for the control signal $u \in \mathcal{U}$ and parameter $\mu$, starting at the initial condition $y(0 ; \mu)=x$. We note that we use the subscript $x$ in $y_{x}$ whenever we want to emphasize the dependence on the initial condition $x$. The above characterization can, under certain regularity assumptions on the value function, be used to derive the HJB equation:

$$
\lambda v(x ; \mu)+\sup _{u \in U}\{-f(x, u ; \mu) \cdot \nabla v(x ; \mu)-g(x, u ; \mu)\}=0, \quad x \in \mathbb{R}^{n},
$$

where $\nabla$ denotes the gradient with respect to $x$ from which the value function can be computed as the unique viscosity solution. We refer to [8] for more details about existence and uniqueness of the value function. The knowledge of the value function allows the computation of the feedback control as follows:

$$
u^{*}(x ; \mu)=\min _{u \in U}\{f(x, u ; \mu) \cdot \nabla v(x ; \mu)+g(x, u ; \mu)\} .
$$


Next, we derive a numerical scheme to approximate the value function $v(x ; \mu)$. For that purpose, we apply an SL scheme to Equation (4) and thus first choose a bounded domain $\Omega \subset \mathbb{R}^{n}$ which we then discretize by a finite set of points $\Xi=$ $\left\{x_{i}\right\}_{i \in J}$ with $J:=\left\{1, \ldots, N_{G}\right\}$ and $N_{G}=|\Xi|$. We address the choice of the domain $\Omega$ and its discretization in Section 3.3. Typically, $\Xi$ is a grid in $n$ dimensions and the number of grid nodes $N_{G}$ grows exponentially with the dimension $n$ such that already coarse discretizations lead to numbers that easily exceed the memory capacities of modern computers. This again highlights the need for MOR techniques for highdimensional problems. We construct a fully discrete SL scheme for the approximate value function which follows from the DPP after temporal discretization of the ODEs for $y$ and a rectangular quadrature rule for the cost functional

$$
V\left(x_{i} ; \mu\right)=\min _{u \in U}\left\{\mathrm{e}^{-\lambda \Delta t} I_{1}[V]\left(x_{i}+\Delta t \Phi\left(x_{i}, u ; \Delta t, \mu\right)\right)+\Delta t g\left(x_{i}, u ; \mu\right)\right\}, i \in J
$$

Here, $V\left(x_{i} ; \mu\right)$ is the approximate value for $v\left(x_{i} ; \mu\right)$ for the nodes of the grid $\Xi$, the constant $\Delta t>0$ denotes the time-step that is used for the temporal discretization, and $\Phi$ is the increment function and includes, for instance, implicit or explicit Euler schemes. Here, $I_{1}[V]$ denotes a first-order interpolant of the discrete value function $V$, e.g., a piece-wise multi-linear interpolation. This is necessary, because the point $x_{i}+\Delta t \Phi\left(x_{i}, u ; \Delta t, \mu\right)$ is usually not a node of the state space grid. Finally, let us exemplify how the increment function $\Phi$, introduced in Equation (5), is chosen when an explicit Euler scheme is performed:

$$
\Phi(x, u ; \Delta t, \mu)=f(x, u ; \mu) .
$$

We refer the reader to [18] for specific details concerning the SL schemes for HJB equations and convergence results in $L^{\infty}$ valid in any dimension.

We are able to approximate a solution to Equation (4) only up to a few dimensions, using the SL discretization with an efficient iterative solver for Equation (5). The simplest algorithm is based on a fixed point iteration of the value function, also called value iteration (VI):

$$
\begin{aligned}
{\left[V^{(j+1)}(\mu)\right]_{i} } & =S\left(\left[V^{(j)}(\mu)\right]_{i}\right), \text { for } j=0,1, \ldots \\
{[S(V)]_{i} } & \equiv \min _{u \in U}\left\{\mathrm{e}^{-\lambda \Delta t} I_{1}[V]\left(x_{i}+\Delta t \Phi\left(x_{i}, u ; \Delta t, \mu\right)\right)+\Delta t g\left(x_{i}, u ; \mu\right)\right\} \quad i \in J .
\end{aligned}
$$

Here, we collect the nodal values in vectors $V^{(j)}(\mu) \in \mathbb{R}^{N_{G}}$, meaning $\left[V^{(j)}(\mu)\right]_{i} \approx$ $V\left(x_{i} ; \mu\right)$ where again the subscript indicates the index $i \in J$ and the superscript $j$ denotes the iteration index. Convergence is guaranteed for any initial guess $V^{(0)} \in$ $\mathbb{R}^{N_{G}}$ since the operator $S: \mathbb{R}^{N_{G}} \rightarrow \mathbb{R}^{N_{G}}$ is a contraction mapping (see, e.g., [17]). Although being simple and reliable, this algorithm is computationally demanding and slow when fine grids are considered.

A more efficient formulation is the so-called policy iteration algorithm (PI, see, e.g., $[25,34]$, which starting from an initial guess $u^{(0)} \in U^{N_{G}}$ of the control at every node, performs the following iterative procedure for $i \in J$

$$
\begin{aligned}
{\left[V^{(j)}\right]_{i} } & =e^{-\lambda \Delta t} I_{1}\left[V^{(j)}\right]\left(x_{i}+\Delta t \Phi\left(x_{i}, u^{(j)} ; \Delta t, \mu\right)\right)+\Delta t g\left(x_{i}, u^{(j)} ; \mu\right), \quad \text { (7) } \\
{\left[u^{(j+1)}\right]_{i} } & =\underset{u \in U}{\operatorname{argmin}}\left\{e^{-\lambda \Delta t} I_{1}\left[V^{(j)}\right]\left(x_{i}+\Delta t \Phi\left(x_{i}, u ; \Delta t, \mu\right)\right)+\Delta \operatorname{tg}\left(x_{i}, u ; \mu\right)\right\}(8)
\end{aligned}
$$


In the first step of (7), the PI method consists of a linear system solve since the control $u^{(j)}$ is fixed and we do not have to compute the minimization problem. Then, the control is updated according to the value function computed in the previous step. We iterate this process until we get the desired accuracy of the value function. It is wellknown (see, e.g., [13, 34]) that the PI algorithm has quadratic convergence provided a good initial guess. This point is very delicate since it requires to know a reasonable approximation of the value function. To solve this problem, we utilize an acceleration mechanism based on a VI solution on a coarse grid, which is used to generate an initial guess for PI on the fine grid, see also Section 3.4. This idea is based on the fact that VI generates a fast error decay when applied over coarse meshes for any initial guess. Thus, we obtain an initial guess close to the solution and we can switch to the PI method over a fine grid, which then converges fast. Therefore, the algorithm is a way to enhance PI with both efficiency and robustness features. We refer to [1] for a detailed description of the algorithm. Finally, we note that in both algorithms, we penalize the value function outside of the numerical domain to impose artificial state constraints boundary conditions as proposed in [17].

The main advantage of the DPP approach presented in this section is the possibility to have a synthesis of feedback controls: Once the value function is computed, the approximated optimal control for a point $x \in \mathbb{R}^{n}$ in the state space is obtained by:

$$
u^{*}(x)=\underset{u \in U}{\operatorname{argmin}}\left\{e^{-\lambda \Delta t} I_{1}[V](x+\Delta t \Phi(x, u ; \Delta t, \mu))+\Delta \operatorname{tg}(x, u ; \mu)\right\} .
$$

For the implementation of this feedback control, a direct search for the minimum can be performed if $U$ contains a finite number of control values. In other scenarios, an efficient minimization algorithm can be employed.

\section{Framework for parametric HJB equations and feedback control}

We now provide details about the use of MOR in the context of DPP in Section 3.1. In particular, we propose a complete and automatic strategy to deal with non-linear parametric feedback control problems via MOR and DPP. From a computational point of view, we also show how the procedure can be implemented efficiently by employing an offline-online splitting of the whole workflow. The overall picture of the procedure is summarized in Fig. 1.

The first step according to Fig. 1 is an adaptive basis generation. By this, we aim at constructing suitable low-dimensional subspaces onto which we project the highdimensional control problem. Details about this step are explained in Section 3.2. For the numerical approximation of the value function of the so-obtained lowdimensional control problems, we perform an SL scheme. To this end, we have to prescribe a finite computational domain on which the SL scheme can be applied. To obtain knowledge about this domain, we propose to make use of a data-driven method to gather statistical information about the distribution of the reduced coordinates, see Section 3.3. The actual calculation of the value function is then performed in two steps: In a precalculation step, we employ a VI scheme to get coarse approximations of the value functions in each parameter subregion, see Section 3.4.1. Furthermore, 


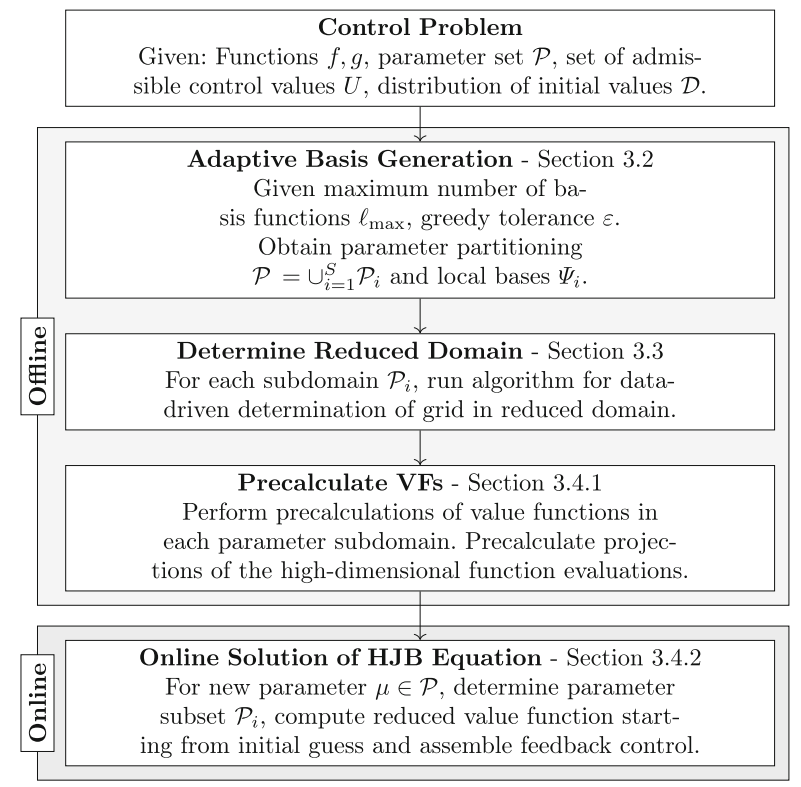

Fig. 1 The workflow from a given parametric control problem towards the online approximation of the value function and feedback control

we precompute several expensive quantities and store them for a later reuse. This concludes the offline step. Online, given a parameter from the parameter domain, we refine the initial guess of the value function from the offline phase with a PI algorithm, see Section 3.4.2. Based on the refined value function, we then define the feedback control that is used to control the full dynamical system. In what follows, we discuss in detail each of the ingredients in Fig. 1.

\subsection{Projection-based approximation of the HJB equation}

The focus of this section is to establish a coupling between MOR and the HJB approach, as initially proposed in [30] for the non-parametric case. The need of MOR is crucial when dealing with high-dimensional problems, such as discretized PDEs, since the curse of dimensionality prohibits a direct solution of the HJB equations in higher dimensions. We apply model reduction for the dynamical system to obtain a reduced system whose dimension then allows to approximate the HJB equation. The ROM is based on projecting the non-linear dynamics onto a low $\ell$-dimensional subspace $\mathcal{V} \subset \mathbb{R}^{n}$ that contains the relevant information about the dynamics $y(t ; \mu)$. We equip the space $\mathcal{V}$ with an orthonormal basis, given by the columns in the matrix $\Psi \in \mathbb{R}^{n \times \ell}$, which will be specified in the following section. We then approximate the full state vector by the linear combination of basis vectors, i.e., $y(t ; \mu) \approx \Psi y^{\ell}(t ; \mu)$ where $y^{\ell}:[0, \infty) \rightarrow \mathbb{R}^{\ell}$ are the so-called reduced coordinates. Plugging this 
ansatz into the dynamical system and requiring a Galerkin condition, we obtain an ODE-system of dimension $\ell$

$$
\begin{aligned}
& \dot{y}^{\ell}(t ; \mu)=\Psi^{T} f\left(\Psi y^{\ell}(t), u(t) ; \mu\right), \quad t>0, \\
& y^{\ell}(0 ; \mu)=\Psi^{T} x .
\end{aligned}
$$

The procedure presented above is a generic framework for MOR for dynamical systems. We note that it is possible to extend the whole procedure performing a Petrov-Galerkin projection and we refer to [5] for a detailed description of the method. It is clear that the quality of the approximation highly depends on the reduced space $\mathcal{V}$, i.e., on the chosen basis $\Psi$. In particular, the dynamical system (9) should capture sufficient information to allow for accurate approximations of the closed-loop behavior for any desired initial state.

To ease the notations, we introduce abbreviations for the reduced quantities. The initial value will be denoted by $x^{\ell}:=\Psi^{T} x$ whereas the projected dynamical system and the reduced running cost function are given as

$$
\begin{aligned}
& f^{\ell}\left(y^{\ell}(t ; \mu), u(t) ; \mu\right):=\Psi^{T} f\left(\Psi y^{\ell}(t ; \mu), u(t) ; \mu\right), \\
& g^{\ell}\left(y^{\ell}(t ; \mu), u(t) ; \mu\right) \quad:=g\left(\Psi y^{\ell}(t ; \mu), u(t) ; \mu\right) .
\end{aligned}
$$

In the general projection framework above, we define the optimal control problem for the projected system:

$$
\begin{aligned}
\inf _{u \in \mathcal{U}} J_{x^{\ell}}^{\ell}(u ; \mu) & :=\inf _{u \in \mathcal{U}} \int_{0}^{\infty} g^{\ell}\left(y^{\ell}(t ; \mu), u(t) ; \mu\right) \mathrm{e}^{-\lambda t} d t, \\
\text { s.t. } \quad \dot{y}^{\ell}(t ; \mu) & =f^{\ell}\left(y^{\ell}(t ; \mu), u(t) ; \mu\right), \quad t>0, \\
y^{\ell}(0 ; \mu) & =x^{\ell} .
\end{aligned}
$$

As in the full-dimensional case, we define the value function for the reduced system

$$
v^{\ell}\left(x^{\ell} ; \mu\right):=\inf _{u \in \mathcal{U}} J_{x^{\ell}}^{\ell}(u ; \mu),
$$

which satisfies the reduced HJB equation which is now $\ell$-dimensional and feasible for numerical treatment as long as the dimension is sufficiently small, e.g., $\ell \leq 5$

$$
\lambda v^{\ell}\left(x^{\ell} ; \mu\right)+\sup _{u \in U}\left\{-f^{\ell}\left(x^{\ell}, u ; \mu\right) \cdot \nabla_{x^{\ell}} v^{\ell}\left(x^{\ell} ; \mu\right)-g^{\ell}\left(x^{\ell}, u ; \mu\right)\right\}=0, \quad x^{\ell} \in \mathbb{R}^{\ell} .
$$

In [4], the authors proved existence and uniqueness of the reduced dynamics $f^{\ell}$. Similarly, one can prove that the reduced HJB equation (12) admits a unique viscosity solution as the original problem since it is obtained by orthogonal projection.

The overall idea is now to replace the high-dimensional value function $v(x ; \mu)$ by its reduced counterpart $v^{\ell}\left(x^{\ell} ; \mu\right)$. Furthermore, we make use of the reduced value function and define the following approximated feedback law, which is essentially the control law from the full-dimensional system, where the value function is replaced by the low-dimensional approximation:

$$
u^{\ell}(x)=\min _{u \in U}\left\{f(x, u ; \mu) \cdot \nabla v^{\ell}\left(x^{\ell} ; \mu\right)+g(x, u ; \mu)\right\} .
$$

In Section 4, we will show the quality of the feedback control, when applied to the full-dimensional system. 
To obtain computationally efficient schemes, we assume that the dynamics has a linear dependence with respect to the control $u$

$$
f(y, u ; \mu)=f^{y}(y ; \mu)+f^{u}(y ; \mu) u,
$$

and that the cost functional has a quadratic form

$$
g(y, u ; \mu)=y^{T} Q(\mu) y+u^{T} R(\mu) u,
$$

where $Q(\mu) \in \mathbb{R}^{n \times n}$ is symmetric positive semidefinite and $R(\mu) \in \mathbb{R}^{p \times p}$ is symmetric positive definite.

\subsection{Basis generation algorithm}

Let us now provide more information about the computation of the basis functions $\Psi$. Since our numerical schemes are limited to a very low number of basis functions, the quality of the basis is of utmost importance. In [5], a comparison for different basis generation techniques in the context of feedback control via the HJB equation is performed in the non-parametric context. It turns out that classical but straightforwad approaches such as POD or BT do not necessarily yield satisfying results. For POD, this is due to the fact that its focus is on providing surrogate models for the dynamics and not for feedback control. For BT, the suboptimality is based on its focus to approximate well input-output transfer, but is not aiming to minimize some optimal control functional. A more robust approach for linear control problems is based on the LQG. The coupling with BT has been studied in $[12,14]$. A different approach that is based on the explicit form of the value function in the linear case is introduced in [5].

Finding the basis functions is of course rather hard for arbitrary non-linear control problems. Unlike for linear problems with quadratic cost functionals where the value function can be computed explicitly by solving an Algebraic Riccati Equation (ARE), the value function for non-linear problems is in general not known analytically. However, we can always obtain local information about the basis by linearizing the control problem around a constant point of interest $(\bar{y}, \bar{u})$ :

$$
f(y, u ; \mu) \approx f_{y}(\bar{y}, \bar{u} ; \mu)(y-\bar{y})+f_{u}(\bar{y}, \bar{u} ; \mu)(u-\bar{u}) .
$$

In the sequel, we will typically choose $(\bar{y}, \bar{u})=0$, since we are interested in steering the system to the origin and hence can write the linearized state equation as

$$
\dot{y}=A(\mu) y+B(\mu) u,
$$

with matrices $A(\mu):=f_{y}(\bar{y}, \bar{u} ; \mu) \in \mathbb{R}^{n \times n}, B(\mu):=f_{u}(\bar{y}, \bar{u} ; \mu) \in \mathbb{R}^{n \times p}$.

Solving the linearized optimal control problem can be easily done by means of the associated discounted ARE for the positive semi-definite and stabilizing solution $P(\mu) \in \mathbb{R}^{n \times n}$

$$
\begin{array}{r}
\mathcal{R}(P(\mu)):=\left(A(\mu)-\frac{\lambda}{2} I_{n}\right)^{T} P(\mu)+P(\mu)\left(A(\mu)-\frac{\lambda}{2} I_{n}\right)- \\
P(\mu) B(\mu) R(\mu)^{-1} B(\mu)^{T} P(\mu)+Q(\mu)=0,
\end{array}
$$


where $\mathcal{R}(P(\mu))$ is the residual of the ARE, and $Q(\mu)$ and $R(\mu)$ are given accordingly in (15). We refer to [18] for a detailed derivation of the ARE under the presence of the discount factor $\lambda$. The idea is now to build basis functions upon the information on the value function from the linearized control problem for varying parameters, although it is just an approximation to the true and unknown value function. For that purpose, we adopt the low-rank factor greedy (LRFG) procedure from [36] to our setting. For the sake of completeness, we summarize the method in Algorithm 1. It runs in a typical greedy structure: An error indicator is minimized over a suitably large but finite training set $\mathcal{P}_{\text {train }} \subset \mathcal{P}$ of parameters by adding information about the worst-approximated true solution in each iteration. In line 4 of the algorithm, only the part which is not yet captured in the basis is considered by orthogonalization and in step 5, the remaining information is compressed via an additional POD, where we prescribe a desired level $1-\varepsilon_{\mathrm{POD}}$ of POD-energy for some $\varepsilon_{\mathrm{POD}} \in[0,1]$ that should be captured by the basis (see, e.g., [21, 36] for details). The algorithm is a variant of the POD-Greedy procedure, which is known to be quasi-optimal for MOR of parametric unsteady PDEs (see, e.g., [22]). We choose the error indicator as the normalized residual norm $\Delta(\mu):=\|\mathcal{R}(\hat{P}(\mu))\|_{F} /\|Q(\mu)\|_{F}$ where $\hat{P}(\mu) \in \mathbb{R}^{n \times n}$ is the approximate solution to the ARE for the current basis. We note that the error indicator proposed here was certified in [36]. This procedure might be expensive since it

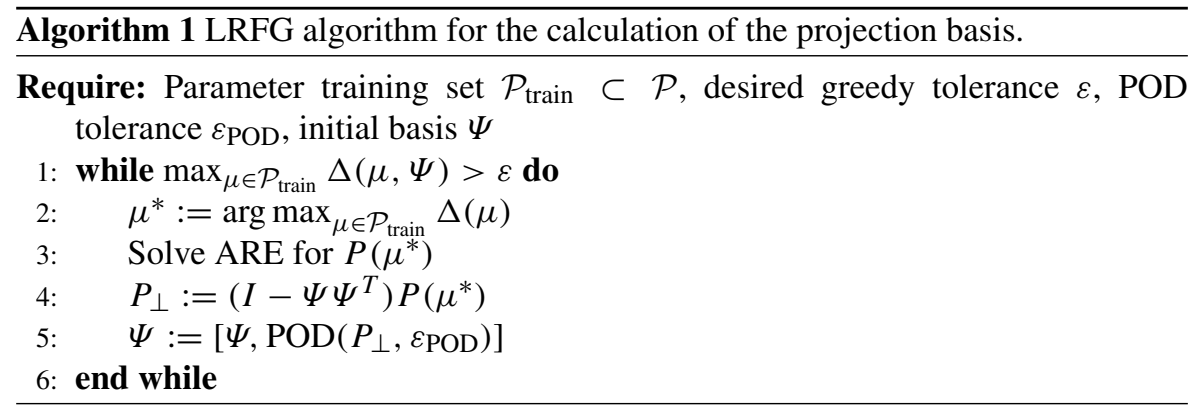

requires the solution of an ARE and a subsequent SVD in each iteration. However, by employing low-rank techniques for the solution of the large-scale AREs, both of these tasks can be sped up substantially. We also refer the interested reader to the recent work [37] for an in-depth discussion of projection-based model reduction for the ARE and the link to the LQR problem. As already mentioned, the strength of this model reduction approach relies on the fact that the basis functions contain directly information of the value function for the infinite horizon problem. Furthermore, the whole described technique does not depend on a particular choice of the control, unlike POD.

By applying a grid-based scheme for the approximation of the value function, we are restricted to a relatively low number of dimensions $\ell$ for which the procedure can be performed. Furthermore, the presence of parameters can change the control problem significantly when going from one configuration to another. Therefore, a basis which is able to capture information about the whole parameter domain might 
easily exceed the maximum possible dimension. To overcome this problem, we apply an adaptive method introducing a partitioning of the parameter domain $\mathcal{P}$. By running the adaptive algorithm, the parameter domain $\mathcal{P}$ is split into $S$ partitions $\mathcal{P}_{i} \subset \mathcal{P}$ such that $\mathcal{P}=\cup_{i=1}^{S} \mathcal{P}_{i}$ together with local bases $\Psi_{i}, \in \mathbb{R}^{n \times \ell_{i}}$ where $\Psi_{i}$ are the $\ell_{i}$ basis functions computed for the partition $\mathcal{P}_{i}$ for $i=1, \ldots, S$.

The idea behind the partitioning is that we want to deal with a prescribed maximum number $\ell_{\max }$ of basis functions for each subregion of the parameter domain to guarantee the computational feasibility of the reduced control problem (11) and, simultaneously guarantee a certain accuracy $\varepsilon$. The algorithm works as follows: Given a partitioning $\left\{\mathcal{P}_{i}\right\}_{i=1}^{S}$ which initially is set to $\mathcal{P}$, we run the basis generation on each parameter subset $\mathcal{P}_{i}$ independently. Two cases can occur: Either the desired accuracy is reached within the prescribed number of basis functions $\ell_{\max }$, or the error indicator/the number of basis functions is too large. In the latter case, the parameter region $\mathcal{P}_{i}$ is refined for example by bisection and the procedure is repeated on all newly identified subregions. The method stops when the desired accuracy is reached and the number of basis elements $\ell_{\max }$ is not exceeded in each subdomain. As stopping criterion, we also include a maximum number of refinements since it is not always possible to reach the amount of basis functions required. In these cases, we accept a reduced basis of lower accuracy that satisfies a strict size constraint $\ell_{i} \leq \ell_{\max }$. We refer to, e.g., [16, 23] and the references therein for more details.

\subsection{Data-driven approximation of the reduced domain}

In this section, we provide details on the procedure to determine the domain for the approximation of the reduced HJB equation (12). Although the reduced HJB equation is defined on the full space $\mathbb{R}^{\ell}$, for numerical reasons, we have to restrict ourselves to a bounded domain $\Omega^{\ell} \subset \mathbb{R}^{\ell}$ and the question arises how a reasonable choice can be made. Note that the design of the reduced domain $\Omega^{\ell}$ is also of great importance for the application in feedback control: By applying the reduced-order feedback control from (13), the projection $\Psi^{T} y_{x}(t ; \mu) \in \mathbb{R}^{\ell}$ of the current state of the controlled system onto the reduced space is required and fed into the reduced value function. Hence, in order to get accurate feedback controls, those projected vectors should be contained in the domain $\Omega^{\ell}$ where the reduced HJB equation is approximated. Finally, the use of a domain requires to impose boundary conditions to the reduced HJB equation. A common choice is the penalization of trajectories which exit the domain (see, e.g., [4]).

A common approach is to choose the domain a priori of the form $\Omega^{\ell}=\left[a_{1}, b_{1}\right] \times$ $\ldots \times\left[a_{\ell}, b_{\ell}\right]$ where $a_{i}<b_{i}, i=1, \ldots, \ell$ are prescribed bounds. However, it is not clear how the values $a_{i}$ and $b_{i}$ can be chosen. In particular, in a parametric scenario, the influence of the parameter can greatly alter the dynamics and thus the projections.

In the current work, we propose a novel strategy that makes use of statistical information about the full and reduced coordinates. For that purpose, we assume that the initial values of the high-dimensional problem follow a prescribed multivariate distribution, which we abbreviate by $x \sim \mathcal{D}$ where $\mathcal{D}$ denotes the chosen probability density. This choice is motivated by the following heuristic observation: In 
cases where the states of the discretized system represent nodal values, e.g., in a FE scheme, the values of neighboring nodes are often of very similar magnitude. This results from phenomena like diffusion or other types of transport of information. In other scenarios, often statistical a priori knowledge of the states that can occur in the application is available, e.g., typical temperatures in a heat transfer application. Note that we have to consider some assumptions on the full states $y \in \mathbb{R}^{n}$, since otherwise their projections $y^{\ell}=\Psi^{T} y$ can lie anywhere in $\mathbb{R}^{\ell}$ and the approximation may be arbitrarily bad.

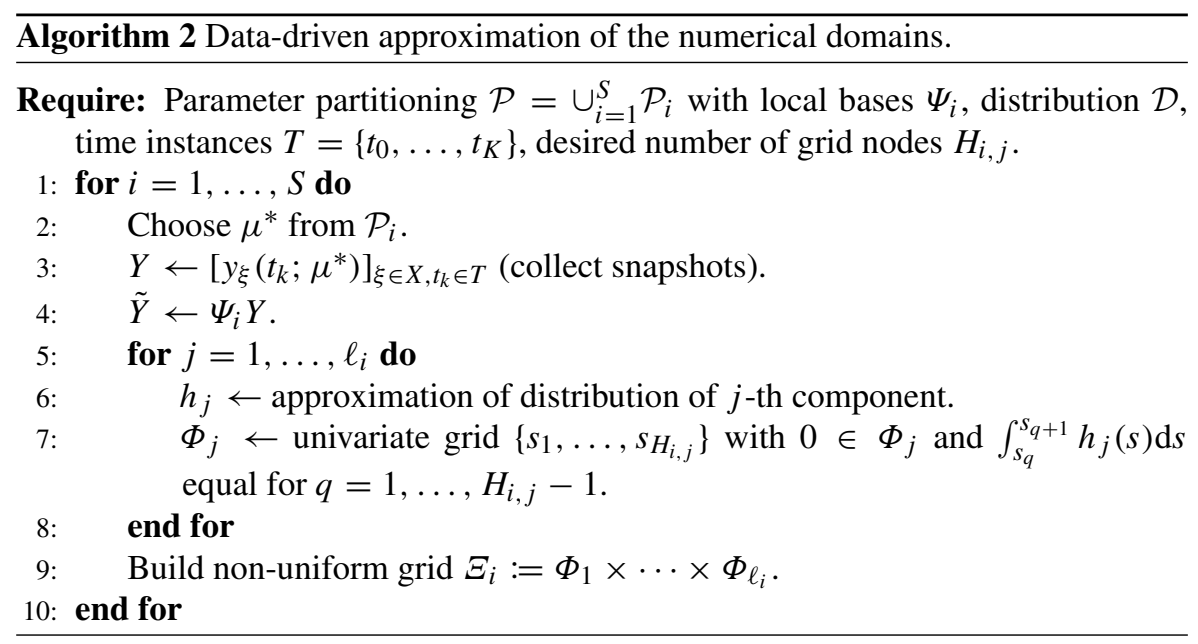

The idea of the proposed algorithm is to sample solutions to the high-dimensional system for certain suitable controls and parameters and to estimate the componentwise distribution of the projected reduced vectors. The algorithm is summarized in Algorithm 2 and illustrated in Fig. 2. Based on the given parameter partitioning, we loop over all $S$ parameter regions and perform the following procedure, where $i$ always denotes the index of the current parameter partition: First, we pick a sample parameter $\mu^{*}$ from the $i$-th parameter domain, e.g., the barycenter and a set of $N_{\text {train }}$ initial conditions $X=\left\{\xi_{1}, \ldots, \xi_{N_{\text {train }}}\right\}$ with $\xi_{k} \sim \mathcal{D}$, for $k=1, \ldots, N_{\text {train. }}$ Then
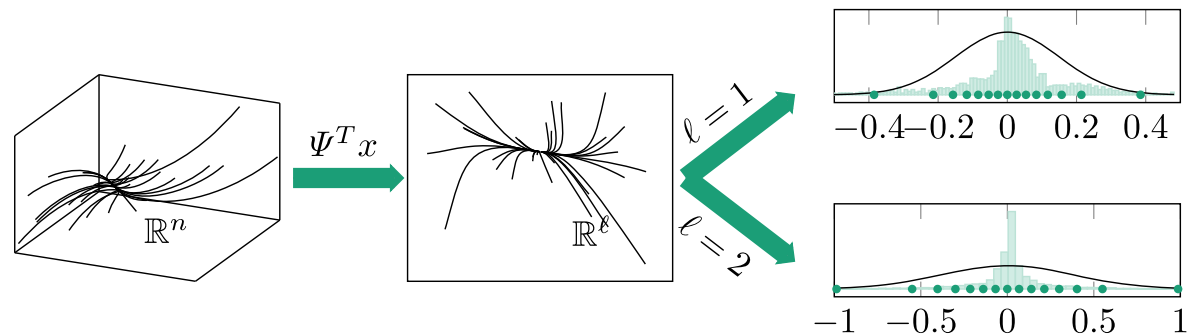

Fig. 2 The procedure for obtaining the statistical distribution and estimating the univariate distributions $(N=15$ partitions in this case $)$ 
we simulate the (controlled) high-dimensional system with the parameter $\mu^{*}$ and all $\xi \in X$ and collect the solution at time instances $T$ in a snapshot matrix $Y$. The control $u^{*}$ can for example be chosen from the linearized system for $\mu^{*}$ or simply be set to zero in case of stable systems. We then project the snapshots onto the $\ell_{i}$-dimensional subspace that is spanned by the basis $\Psi_{i}$ and analyze the result componentwise: To this end, we normalize the data and fit a Gaussian to the distribution of the values in the reduced coordinate. From this, we get a continuous function $h_{j}: \mathbb{R} \rightarrow[0,1]$ with $\int_{\mathbb{R}} h_{j}(s) \mathrm{d} s=1$ which we use to construct a set of grid nodes $\Phi_{j}$ for the component. Given a desired odd number of grid nodes $H_{i, j}$, we enforce the area under the curve of $h_{j}$ to be equal between all grid nodes. By this, we ensure a distribution of the grid nodes that fits to the estimated statistical information. The final grid $\Xi_{i}$ for the parameter region $\mathcal{P}_{i}$ is then defined as the cartesian product of all onedimensional grids and consists of $\left|\Xi_{i}\right|=\prod_{j=1}^{N_{i}} H_{i, j}$ points. A schematic drawing of the procedure for the reduction of $n=3$ to $\ell=2$ is given in Fig. 1 .

\subsection{Offline-online efficient implementation of the numerical scheme}

In this section, we provide some remarks about technical features of the method to improve its computational efficiency. In particular, we will explain how to deal with an offline-online decomposition that is often used in MOR to distinguish the first phase characterized by potentially expensive computations to build a surrogate model (offline stage), which enables rapid and inexpensive simulations (online stage). In the current work, the role of model reduction is slightly different, in fact we are interested in reducing the dimension of the dynamical systems to decrease the complexity of the corresponding HJB equation and, therefore, to make the problem feasible. We do not aim at real-time computations since the method will still rely on the approximation of a high-dimensional PDE. However, by assuming a special structure of the ODE function $f$ and the running cost $g$, we can realize a speed-up since the expensive evaluations of the non-linear function $f$ can be shifted to the offline stage. We recall that an offline-online decomposition, in this context, is new and we will show the computational benefit in Section 4. We further assume that the dynamics given in (14) satisfies the following parameter separability assumption:

$$
f(y, u ; \mu)=f^{y}(y ; \mu)+f^{u}(y ; \mu) u=\sum_{q=1}^{Q_{y}} \Theta_{q}^{y}(\mu) f_{q}^{y}(y)+\sum_{q=1}^{Q_{u}} \Theta_{q}^{u}(\mu) f_{q}^{u}(y) u,
$$

where the functions $\Theta_{i}^{y}, \Theta_{j}^{u}: \mathcal{P} \rightarrow \mathbb{R}$ for $=1, \ldots, Q_{y}$ and $j=1, \ldots, Q_{u}$ are coefficient functions depending only on the parameter $\mu$. This structure allows for the precomputation of most function evaluations that are needed during the online phase.

\subsubsection{Offline stage}

The offline stage constitutes the building block of our approach, where most of the quantities are precomputed and stored for any parameter configuration. It basically consists of three parts: 
1. basis generation, including the parameter partitioning,

2. sampling of the set for the estimation of the grid in the reduced space,

3. preparatory tasks for a fast online PI, which is explained in the following.

We assume that we are given a parameter partitioning $\mathcal{P}_{i}$ for $i=1, \ldots, S$, together with corresponding grids generated by the procedure explained in Section 3.3. Let us denote the corresponding grid nodes corresponding to the $i$ th subdomain $\mathcal{P}_{i}$ as $\Xi_{i}$ with $\left|\Xi_{i}\right|=H_{i}$. In order to speed up the online calculations for the PI, we make use of the special structure defined in Equation (17) and precompute all function evaluations and their projections

$$
\begin{aligned}
& f_{q, i}^{y, \ell}:=\left[\Psi_{i}^{T} f_{q}^{y}\left(\Psi_{i} x_{1}\right), \ldots, \Psi_{i}^{T} f_{q, i}^{y}\left(\Psi_{i} x_{H_{i}}\right)\right], \quad q=1, \ldots, Q_{y}, \\
& f_{q, i}^{u, \ell}:=\left[\Psi_{i}^{T} f_{q}^{u}\left(\Psi_{i} x_{1}\right), \ldots, \Psi_{i}^{T} f_{q, i}^{u}\left(\Psi_{i} x_{H_{i}}\right)\right], \quad q=1, \ldots, Q_{u},
\end{aligned}
$$

where $x_{j} \in \Xi_{i}$ for $j=1, \ldots, H_{i}$ and $i=1, \ldots, S$. Note that the precalculated quantities are of low-dimension and can all be precomputed once in the offline phase. Given a parameter $\mu$, and using assumption (17) the function can be rapidly evaluated on the grid nodes for this parameter by summing up the parameter-independent quantities $f_{q, i}^{y, \ell}$ and $f_{q, i}^{u, \ell}$, weighted by the corresponding coefficient functions $\Theta_{q}^{y}(\mu)$ and $\Theta_{q}^{u}(\mu)$.

This allows us to reduce drastically the number of evaluations of the dynamical system with powerful speed-up in both the VI and PI method since the computation of the value functions involves several evaluations of the dynamical systems. Similar assumptions may be posed on the cost functional. We finally note that (17) is absolute crucial to perform fast evaluations and can be always obtained via the EIM algorithm (see e.g. [9]).

The next step concerns the precalculation of the value function via a VI scheme. As described in Section 2 we solve the reduced HJB equation (12) performing a VI algorithm and then switching to a PI method to obtain fast convergence of the method. We propose to use the VI method offline for some particular choices of the parameter. In fact, since we act with a partition of the parameter domain we assume that in each subregion the dynamics will not differ significantly: since the basis generation yielded a low-dimensional basis we compute an approximation of the value function for the barycenter of each subdomain $\mathcal{P}_{i}$. Therefore, we obtain accurate initial guesses for the value function and guarantee fast convergence when switching to the PI algorithm on a finer grid. Finally, we note that at this stage we compute the finer grid, on which we later use the PI method and evaluate all the quantities independent from the parameter $\mu$ as e.g. $f_{q}^{y, \ell}, f_{q}^{u, \ell}$.

\subsubsection{Online stage}

The precomputation in the offline stage allows us to focus on the following steps in the online phase: Given a new parameter $\mu \in \mathcal{P}$ we have to

1. identify the parameter partition $\mathcal{P}_{i}$ such that $\mu \in \mathcal{P}_{i}$,

2. calculate an accurate approximation for the reduced value function $v^{\ell}(\cdot ; \mu)$, 
3. define the feedback control $u^{\ell}(x)$ according to equation (13).

The first step is trivial and the last step can be readily performed once the approximation $v^{\ell}(\cdot ; \mu)$ is available. For the second step we run a PI algorithm starting from the initial guess for the value function, which was calculated during the offline phase. Note that at this point we can make use of the precalculated function evaluations on the grid $\boldsymbol{\Xi}_{i}$ to speed up the calculation significantly. By doing this, the overall complexity does not depend on the high-dimension $n$ but only on the reduced dimension $\ell_{i}$ and the number of grid points in $\Xi_{i}$. We compute the reduced value function $v^{\ell}(\cdot ; \mu)$ satisfying (12) at each grid point $x^{\ell_{i}} \in \Xi_{i}$.

$$
u^{\ell_{i}}(x ; \mu):=\arg \min _{u \in U}\left\{f(x, u ; \mu) \cdot \nabla_{x^{\ell_{i}}} v^{\ell_{i}}\left(x^{\ell} ; \mu\right)+g(x, u ; \mu)\right\} .
$$

We note that here we replace the high-dimensional value functional with the reduced approximation whereas the dynamics $f$ and the cost functional $g$ are kept high-dimensional due to the fact that the basis functions better describe the value function rather than the dynamics. This strategy turns out to be more stable than using the reduced functions $f^{\ell}$ and $g^{\ell}$.

\section{Numerical tests}

We now present three examples of optimal feedback control problems, which demonstrate the efficiency of our proposed method. The first example models a control problem for a linear advection-diffusion equation where the true optimal feedback control and the true value function can be computed by means of the ARE. Thus, we are able to compare the numerical approximation obtained from our approach to the true solution. The second example is a two-dimensional semi-linear heat equation with a cubic non-linearity which presents an unstable equilibrium around the origin. The control objective will be the stabilization around this point. The last example deals with a coupled viscous Burgers system, introducing many layers of additional complexity since the dimension of the control space is two as well as the number of outputs. Furthermore, the equations for this scenario are described by two coupled non-linear PDEs. The aim of the second and third example is to show that non-linear feedback control is more efficient than a LQR controller based on the linearization of the problem which is then plugged into the non-linear model under consideration.

To apply the workflow, we have to assume certain statistical properties of the highdimensional solution. Since our examples stem from semi-discretized PDEs, we can define those properties based on the nodal values of the discretization. To this end, let $N_{1}, \ldots, N_{n} \in \mathbb{R}^{d}$ be the coordinates of the nodes in either the FE mesh or in the FD discretization, where $d$ is the number of dimensions of the original PDE problem. We then define the Gaussian distribution $\mathcal{D}:=\mathcal{N}(v, \Sigma)$ with mean $v \in \mathbb{R}^{n}$ and positivedefinite covariance matrix $\Sigma \in \mathbb{R}^{n \times n}$. To model the relationship between the nodes we define the entries in the matrix $\Sigma$ as

$$
\Sigma_{i, j}=c \cdot b\left(N_{i}\right) b\left(N_{j}\right) \mathrm{e}^{-\gamma\left\|N_{i}-N_{j}\right\|^{2}}, \quad i, j=1, \ldots, n,
$$


for $\gamma, c>0$. By changing $\gamma$, we adjust the weight of nodes that are close/far to each other. Furthermore, through the function $b: \mathbb{R}^{d} \rightarrow[0, \infty)$, we get the possibility to put different weights on nodes that are, for example, close to the boundary. By this, we can incorporate zero boundary conditions. Figure 3 shows three random vectors drawn according to the distribution $\mathcal{D}$ for a discretization of the interval $[0,1]$ into $n=20$ nodes with different values for $\gamma$, different weight functions $b$ and $v=0$. The example shows the great flexibility in the modeling of the high-dimensional states. In order to support the reproducibility of the results, we provide the MATLAB code online at https://www.ians.uni-stuttgart.de/ anm/research/software/HJBMORFeedback.zip, which is an add-on to the RBmatlab release 1.16.09, which can be obtained at https://www.morepas.org/software/ rbmatlab/

\subsection{Test 1: two-dimensional linear advection-diffusion}

The first test problem considers an optimal control scenario for a two-dimensional linear advection-diffusion equation on the domain $\Omega:=(0,1)^{2}$. The parametric PDE for this example is given by

$$
\begin{aligned}
\partial_{t} w(t, \xi ; \mu)-\mu_{\text {diff }} \Delta w(t, \xi ; \mu)+a(\xi ; \mu) \cdot \nabla w(t, \xi ; \mu) & =1_{\Omega_{B}}(\xi) u(t), \quad \xi \in \Omega, t \geq 0 \\
w(0, \xi ; \mu) & =w_{0}(\xi ; \mu) .
\end{aligned}
$$

The velocity is defined as the divergence-free field at $\xi=\left(\xi_{1}, \xi_{2}\right) \in \Omega$ as

$$
a(\xi ; \mu)=\mu_{\mathrm{adv}} \cdot\left(-\left(\xi_{2}-0.5\right),\left(\xi_{1}-0.5\right)\right)^{T},
$$

which induces a counterclockwise flow in the solution with velocity $\mu_{\mathrm{adv}}$ as shown in Fig. 4a. The indictor function $1_{\Omega_{B}}(\xi)$ maps the scalar control $u(t)$ onto the domain $\Omega_{B}:=[0.5,0.9]^{2}$. The parameters in this example are $\left(\mu_{\text {diff }}, \mu_{\text {adv }}\right)^{T} \in \mathcal{P}:=$ $[0.05,0.1] \times[2,4]$. In order to set up the control problem, we define the standard quadratic cost functional

$$
J_{w_{0}}(u ; \mu):=\int_{0}^{\infty}\left(10 s(t ; \mu)^{2}+10^{-2} u(t)^{2}\right) \mathrm{e}^{-\lambda t} \mathrm{~d} t
$$
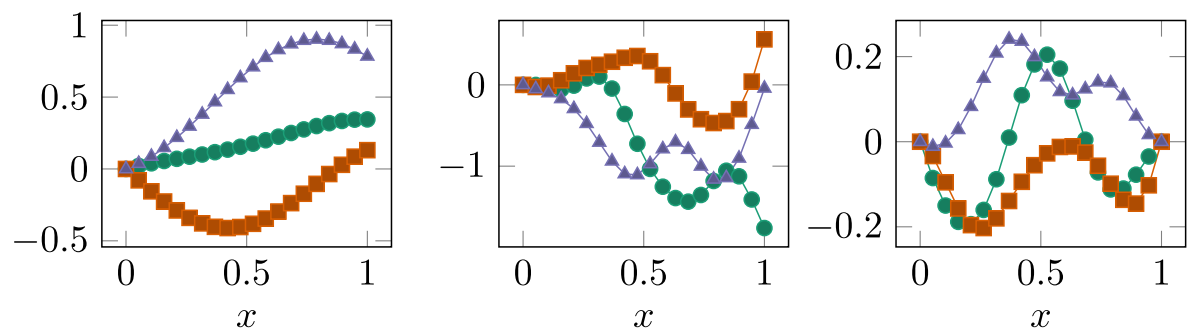

Fig. 3 Three random initial conditions chosen by the distribution $\mathcal{N}$ for different $\gamma$ and $b(\cdot)$ choices: $\gamma=1, b(x)=x($ left $) ; \gamma=20, b(x)=x($ middle $) ; \gamma=10, b(x)=-\frac{1}{4}+\left(x-\frac{1}{2}\right)^{2}$ 


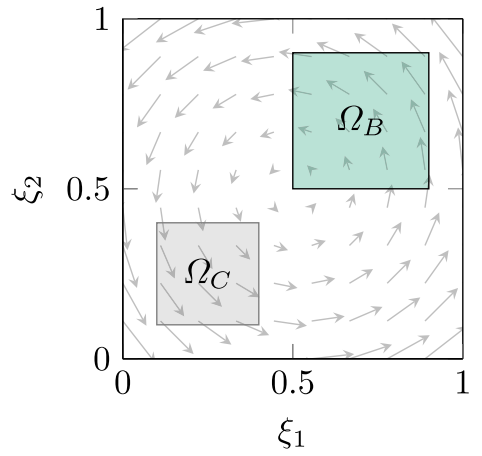

(a) Schematic drawing of the setting.
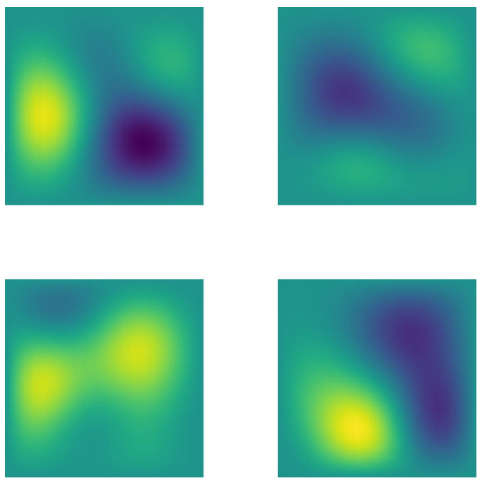

(c) Four random initial values, sampled according to the distribution $\mathcal{N}(0, \Sigma)$.

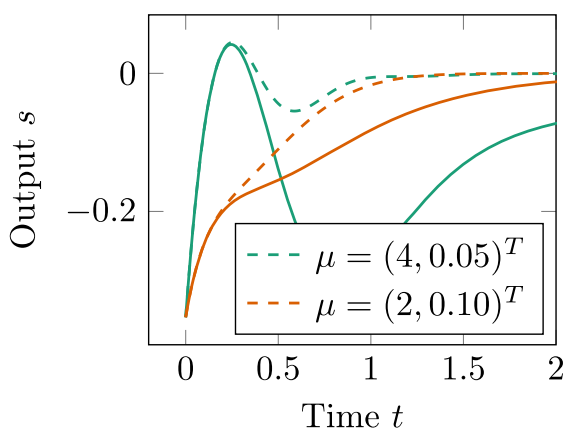

(b) LQR-controlled (dashed) and uncontrolled (solid) outputs for a fixed initial configuration and two different parameters.
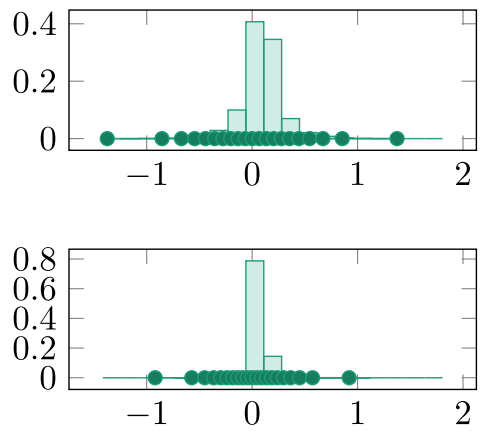

(d) Normalized histogram for the first and second reduced components.

Fig. 4 Linear setting

with

$$
s(t ; \mu):=\frac{1}{\left|\Omega_{C}\right|} \int_{\Omega_{C}} w(t, \xi ; \mu) \mathrm{d} \xi, t \geq 0,
$$

where $\lambda=10^{-3}$ and $\Omega_{C}:=[0.1,0.4]^{2}$. Here, we introduce the quantity of interest $s(t ; \mu)$ that depends on the solution of the PDE $w(\cdot, \cdot ; \mu)$. Figure $4 \mathrm{~b}$ shows the outputs for two different configurations for the controlled and uncontrolled problem. We see how different parameters lead to different outputs. We discretize the control problem in space by using linear finite elements on a uniform triangular grid, resulting in an $n=676$ dimensional LTI system with the scalar discretized output $z$

$$
E \dot{y}=\left(\mu_{\mathrm{diff}} A_{\mathrm{diff}}-\mu_{\mathrm{adv}} A_{\mathrm{adv}}-A_{\text {dirichlet }}\right) y+B u, \quad z=C y, \quad y(0)=x .
$$

We note that Equation (19) fits into the abstract setting shown in (1) with $Q=$ $10 C^{T} C, R=10^{-2}$ in (15) and that $A_{\text {diff }}, A_{\text {adv }}$, and $A_{\text {dirichlet }}$ are the discretization of $\Delta w, \nabla w$ and the boundary conditions, respectively. The temporal discretization is 
carried out with an implicit Euler scheme with step size $\Delta t=10^{-2}$. In what follows, we compare the controlled dynamics with both the HJB and LQR approach. For this setting, it is known that the true value function is given as $v(y ; \mu)=y(0)^{T} P(\mu) y(0)$ and the true optimal control takes the form $u(t)=-K(\mu) y(t)$ with the feedback gain matrix $K(\mu):=R^{-1} B^{T} P(\mu)$ that depends on the solution $P(\mu) \in \mathbb{R}^{n \times n}$ of the ARE (16). To apply the HJB approach, we restrict the control values to the finite set of points $U:=\left\{u^{3} \mid u=-2+i \Delta u, i=0, \ldots, 109\right\}$ where $\Delta u=\frac{4}{109}$, which provides enough information to capture the LQR control values sufficiently accurate.

For all simulations, we choose initial values that are sampled via a Gaussian distribution with covariance matrix (18) with zero mean and the choices with $c=$ $10^{-3}, \gamma=2$ and $b(y):=\left(-4\left(y_{1}-0.5\right)^{2}+1\right)\left(-4\left(y_{2}-0.5\right)^{2}+1\right)$ for the node coordinates $y:=\left(y_{1}, y_{2}\right)^{T} \in \mathbb{R}^{2}$ of the FE mesh. In Fig. $4 \mathrm{c}$ we provide a plot of four random initial vectors, drawn by using the distribution $\mathcal{N}(0, \Sigma)$.

According to our proposed method, we first run the adaptive LRFG algorithm to produce a partition, $\mathcal{P}=\cup_{i=1}^{S} \mathcal{P}_{i}$ of the parameter space and corresponding local bases. The basis generation was performed with a desired tolerance of $\varepsilon=0.9$, maximum basis size 5 and maximum refinement level 3, resulting in a grid as indicated in Fig. 5. The next step consists of gathering statistical information for building the grids in each parameter subregion $\mathcal{P}_{i}$. For that purpose, we run 100 uncontrolled simulations and collect the reduced state vectors. Figure $4 \mathrm{~d}$ shows the distribution of the uncontrolled solutions for the parameter subdomain for $\mu=\left(\mu_{\text {diff }}, \mu_{\text {adv }}\right)^{T}$. We can see that the distribution allows to compute a non-uniform grid which is finer where the distribution is higher. The domain for the reduced HJB equation is computed as discussed in Section 3.3.

Let us first investigate the performance of the HJB approach compared with the true LQR for a fixed parameter. For this purpose, we choose the test parameter $\mu^{*}=$ $(3,0.08)^{T}$ which leads to a non-trivial configuration due to the large advection and small diffusion. We calculate a fixed basis by solving the ARE for this parameter and using the first $\ell=\{1,2,3,4,5\}$ left singular vectors as basis elements, see also Section 3.2. We show the results of the controlled problem and compare the error in the costs of the full-dimensional system steered with the LQR control and the approximated HJB control in Table 1. For that purpose, we pick initial vectors from a test set

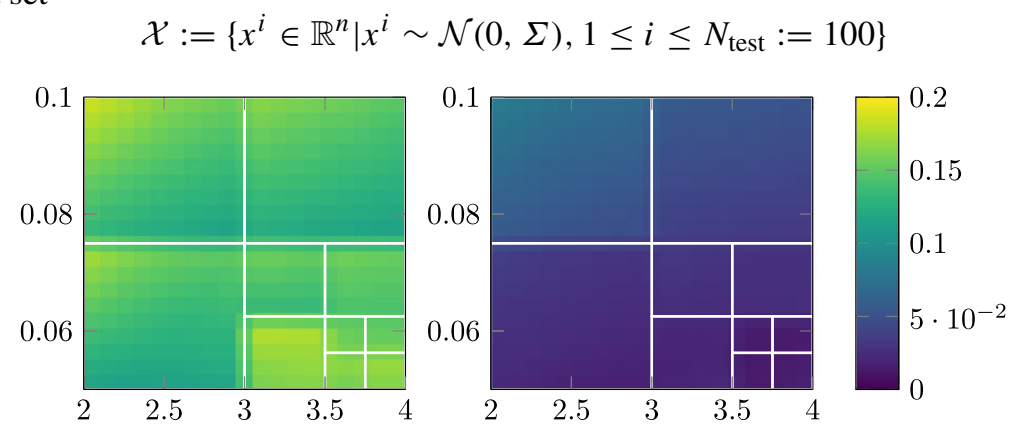

Fig. 5 Test 1: Relative error of the value function computed with VI algorithm in the barycenter of each parameter subregion in the offline stage (left), relative error of the value function computed with a PI algorithm over the whole parameter space in the online stage (right) 
Table 1 Test 1: Approximation error for the fixed parameter $\mu^{*}=(3,0.08)^{T}$

\begin{tabular}{lllllll}
\hline & & \multicolumn{2}{l}{ HJB with 31 points } & & \multicolumn{2}{l}{ HJB with 11 points } \\
\cline { 3 - 4 } \cline { 6 - 7 }$\ell$ & LQR & Equi. & Non-equi. & & Equi. & Non-equi. \\
\hline 1 & $1.18 \cdot 10^{0}$ & $1.98 \cdot 10^{3}$ & $1.10 \cdot 10^{3}$ & & $2.27 \cdot 10^{3}$ & $1.72 \cdot 10^{3}$ \\
2 & $4.34 \cdot 10^{1}$ & $4.69 \cdot 10^{2}$ & $3.92 \cdot 10^{2}$ & & $3.86 \cdot 10^{2}$ & $5.87 \cdot 10^{2}$ \\
3 & $2.53 \cdot x 10^{-1}$ & $1.79 \cdot 10^{-1}$ & $1.42 \cdot 10^{-1}$ & & $2.36 \cdot 10^{-1}$ & $1.78 \cdot 10^{-1}$ \\
4 & $2.12 \cdot 10^{-2}$ & $5.25 \cdot 10^{-2}$ & $2.87 \cdot 10^{-2}$ & & $1.02 \cdot 10^{-1}$ & $6.90 \cdot 10^{-2}$ \\
5 & $2.76 \cdot 10^{-3}$ & $4.23 \cdot 10^{-2}$ & $2.07 \cdot 10^{-2}$ & & $9.27 \cdot 10^{-2}$ & $5.10 \cdot 10^{-2}$ \\
\hline
\end{tabular}

and run the full dimensional simulations with the controls obtained by a reduced LQR controller, e.g., the optimal control law of the reduced order LTI system, and the approximated HJB controls. As error measurement, we define the mean relative error in the costs of the approximated controlled systems $J_{x}(u ; \mu)$ compared to the true LQR cost $J_{x}^{\mathrm{LQR}}(u ; \mu)$ for the initial state $x$ as

$$
\operatorname{mean}_{x \in \mathcal{X}} \frac{\left|J_{x}(u, \mu)-J_{x}^{\mathrm{LQR}}(u, \mu)\right|}{\left|J_{x}^{\mathrm{LQR}}(u, \mu)\right|} .
$$

The first column in Table 1 represents the dimension of the reduced problem. The second column is the error when using the LQR controller which is obtained by solving the ARE for the reduced order system. The third, fourth, fifth, and sixth column show the error between the true value function and value function computed by the HJB approach with 31 points in each dimension (third and fourth column) and only 11 points (fifth and sixth column). As one can see, the reduced problem of dimension 1 and 2 is not stable for both, the LQR and HJB approach. Then increasing the dimension $\ell$, the error decays as expected. It is also possible to see that our proposed approach for the discretization of the reduced domain performs better than the equidistant grid. This is a consequence of a finer grid around the point of interest. We also note that our results are very close to the LQR which we consider optimal here. However, it is hard to make a fair comparison between the LQR and the HJB approach because their settings are not the same, e.g., the control space and the numerical domain. Furthermore, the table shows the quality of the basis functions for a strong advection dominated problem.

Then, let us draw our attention to the parametrized problem. As discussed in Section 3.2 we have to use an adaptive strategy not to exceed a certain number of basis functions to be able to solve the reduced HJB equation. Figure 5 shows how the algorithm identifies subregions in the parameter space. It is somehow intuitive that advection dominated problems need more information on the basis functions and, therefore, further refinements towards higher $\mu_{\mathrm{adv}}$ and lower $\mu_{\text {diff. Here, we decide }}$ to use $\ell=4$ basis functions in each subregion. Finally, we show the error over the whole parameter space in Fig. 5. In the left panel one can see the error of the value function computed with an offline VI algorithm only in the barycenter of each subregion. In the right panel, we see how the PI algorithm improves the approximation of 
the value functions. We also note that the VI is computed on a very coarse grid with 9 points in each dimension, whereas the PI algorithm is computed with 25 points. This plot also shows the benefit of the offline-online decomposition in terms of accuracy of the value function.

\subsection{Test 2: non-linear unstable 2D heat equation}

The second test problem deals with the control of a two-dimensional semi-linear advection-diffusion equation with a cubic non-linearity on the domain $\Omega:=(0,1)^{2}$. The parametric PDE is defined as

$$
\begin{aligned}
& \partial_{t} w(t, \xi ; \mu)-\mathcal{L} w(t, \xi ; \mu)+\mu\left(w(t, \xi ; \mu)-w(t, \xi ; \mu)^{3}\right)=1_{\Omega_{B}}(\xi) u(t), \\
& \xi \in \Omega, t \geq 0 \text {, }
\end{aligned}
$$

where the linear operator $\mathcal{L} w:=0.2 \Delta w-\nabla \cdot w$ describes the diffusion and advection part. We impose homogeneous Dirichlet boundary conditions on all boundaries and define the distributed control input via the indicator function $1_{\Omega_{B}}(\xi)$ on the domain $\Omega_{B}:=[0.2,0.6]^{2}$. The parameter $\mu$ directly influences the strength of the cubic nonlinearity and takes values in the set $\mathcal{P}:=[2,7]$. We consider $Q=10, R=1$ and the discount factor is $\lambda=10^{-3}$ in (15). The problem is spatially discretized by using a finite difference scheme on a uniform grid with $n=361$ nodes. The resulting system takes the form $\dot{y}=A y+\mu F(y)+B u$, which fits to our assumption about the offlineonline splitting for the online PI. Here, the non-linearity $F(y)$ is the componentwise evaluation of the cubic non-linearity, e.g., $(F(y))_{i}=\left(y_{i}-y_{i}^{3}\right)$. The temporal discretization is performed by applying an explicit Euler scheme with step size $\Delta t=$ $10^{-3}$. Equation (20) has 3 equilibria, where $w=0$ is unstable. The uncontrolled dynamics reach either the stable equilibrium depicted in Fig. 6a or the one which has the same structure but opposite sign. The control goal is to steer the solution to the unstable origin and keep it there. We also note that this particular example is not stable under finite-time open-loop control, since it is impossible to reach exactly zero and any small deviation will lead to instabilities. We also mention that a model predictive control approach can be applied as an alternative to our proposed method, see e.g. [20].

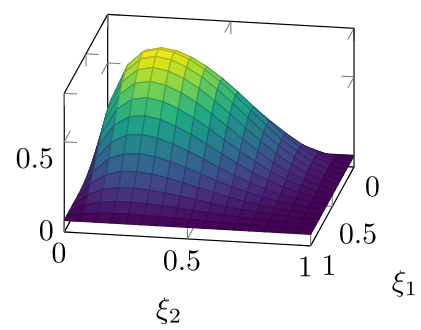

(a) Equilibrium state.

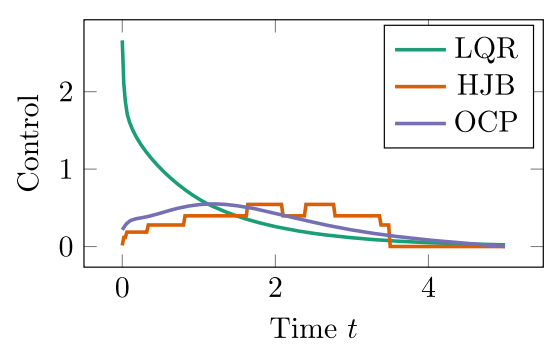

(b) Control functions for the same initial configuration.

Fig. 6 Test 2: Equilibrium state and control values for $\mu=7$ 
For the sake of completeness we also show the control input in Fig. 6b computed with the LQR, the HJB and an open-loop approach. We note that the HJB approach agrees with the open-loop solution. However, the latter method is computationally very expensive since we have to chose a long horizon to approximate the infinite horizon problem and this might lead to unstable solutions. For these reasons, in what follows, we only compare our results with the LQR routine. We recall that the LQR controller is computed from the linearized system and plugged into the non-linear model. In this example, we again make use of a Gaussian distribution with covariance matrix (18) with $b$ chosen as in the linear and with $\gamma=5$ and $c=0.45$.

We apply the procedure proposed in this paper and, therefore, start with the basis generation. The linearization around the origin of the state equation yields an LTI system of the form $\dot{y}=\left(A+\mu I_{n}\right) y+B u$. Applying the adaptive LRFG algorithm for this example is not trivial because the solutions to the ARE do not have a lowrank structure but full numerical rank. A heuristic explanation for this is that we measure the full state in the cost functional instead of an output of interest. However, we successfully apply the algorithm and prescribe a desired level of refinement and run the algorithm. The algorithm then refines uniformly over the parameter space up to the prescribed level. We run this procedure with maximum refinement levels 1 and 3 , resulting in two and 16 partitions.

In Fig. 7 we show the average ratio $\operatorname{mean}_{x \in \mathcal{X}} J_{x}^{L Q R} / J_{x}^{H J B}$ for 100 random samples to demostrate the improvement of the HJB approach over using a classical LQR controller obtained from the linearized system. As one can see, for small values of the parameter $\mu$, our results are very close to the LQR setting due to a small contribution of the non-linear term. However, when increasing $\mu$, we can observe a huge improvement with the HJB approach. Furthermore, we see how the refined parameter partitioning influences the accuracy of our approximations. Even though both refinement levels yield the same improvement over the LQR-controlled simulation after running an online PI, we see that we are able to reach the same quality of approximation by just using the value functions calculated offline in the barycenters for the third refinement case. From a computational point of view, we note that the online PI starting from the coarse approximations with one refinement level requires much more iterations to converge.

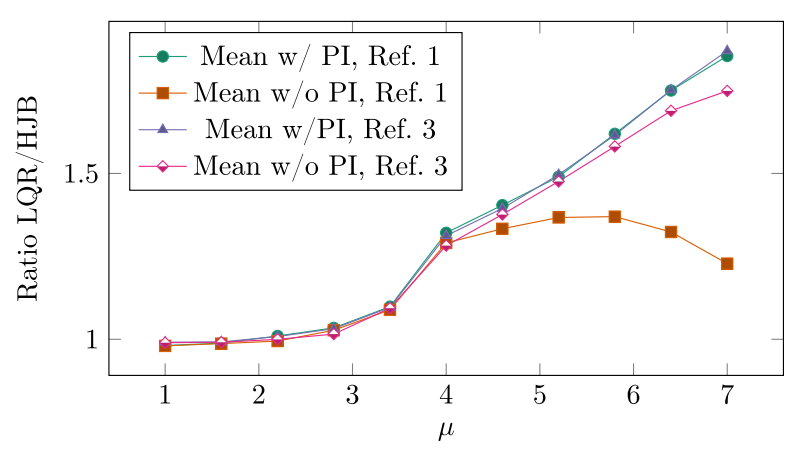

Fig. 7 Test 2: Plot showing the improvement of the HJB approach vs. a classical LQR approach 


\subsection{Test 3: burgers system}

We consider the following two-dimensional coupled Burgers equations for $\xi \in \Omega$ : $=(0,1)^{2}$ and $t \geq 0$

$$
\begin{aligned}
\partial_{t} w(t, \xi ; \mu)-\sigma \Delta w(t, \xi ; \mu)+(w(t, \xi ; \mu) \cdot \nabla) w(t, \xi ; \mu) & =1_{\Omega_{B}}(\xi) u(t), \\
w(0, \xi ; \mu) & =w_{0}(\xi ; \mu),
\end{aligned}
$$

for the unknown function $w(t, \xi ; \mu)=\left(w_{1}(t, \xi ; \mu), w_{2}(t, \xi ; \mu)\right)^{T} \in \mathbb{R}^{2}$. We impose homogeneous Dirichlet boundary conditions on all boundaries and choose a low diffusion constant $\sigma=10^{-4}$. The indicator function $1_{\Omega_{B}(\xi)}$ maps the two control functions $u(t)=\left(u_{1}(t), u_{2}(t)\right)^{T}$ onto the subdomain which is given, componentwise, by the ball of radius 0.2 centered in $(0.5,0.25)^{T}$, i.e. $\Omega_{B}=B_{0.2}\left((0.5,0.25)^{T}\right)$. We consider two partial measurements $s_{1}(t), s_{2}(t)$ from the system:

$$
s(t ; \mu):=\left(\mu_{1} \int_{\Omega_{C}} w_{1}(t, \xi ; \mu) \mathrm{d} \xi, \quad \mu_{2} \int_{\Omega_{C}} w_{2}(t, \xi) \mathrm{d} \xi\right), \quad t \geq 0,
$$

which are the average velocities of the flow in $\xi_{1}$ and $\xi_{2}$ direction, measured on the subdomain $\Omega_{C}:=B_{0.2}\left((0.5,0.25)^{T}\right)$ and the parameters $\mu_{1}, \mu_{2} \in \mathcal{P}:=[0.01,5]$ determine weights on the individual flow components. The cost functional for the PDE control problem is given by:

$$
\int_{0}^{\infty} \mathrm{e}^{-\lambda t}\left(\|s(t ; \mu)\|^{2}+\|u(t)\|^{2}\right) \mathrm{d} t
$$

with the discount factor $\lambda=10^{-4}$. We discretize the system (21) by a finite difference scheme with an upwind flux for the convection term which leads to a system of ODEs of dimension $n=800$

$$
\dot{y}(t)=f(y(t), u(t))=\sigma A y(t)+B u(t)+F(x(t)), \quad z(t ; \mu)=C(\mu) y(t),
$$

where the discretized output $z(t)$ stems from a discretization of Equation (22) by a rectangular quadrature rule. Since we consider two inputs and two outputs, the dimension of the matrices are given as $B \in \mathbb{R}^{n \times 2}$ and $C(\mu) \in \mathbb{R}^{2 \times n}$. As control space we choose $U=\bar{U}^{2}$ where $\left.\bar{U}=\left\{u^{3} \mid u=-3+0.1875 i, i=0, \ldots, 32\right\}\right\}$. The temporal discretization is carried out with an explicit Euler scheme with time
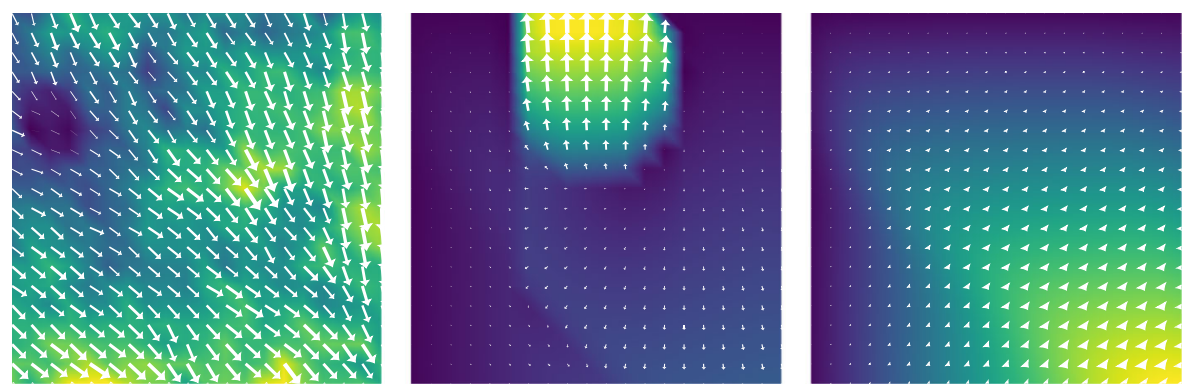

Fig. 8 Test 3: Example of the dynamical behaviour of the Burgers equation. From left to right: Initial state, controlled state and uncontrolled state at $t=2.5$ 


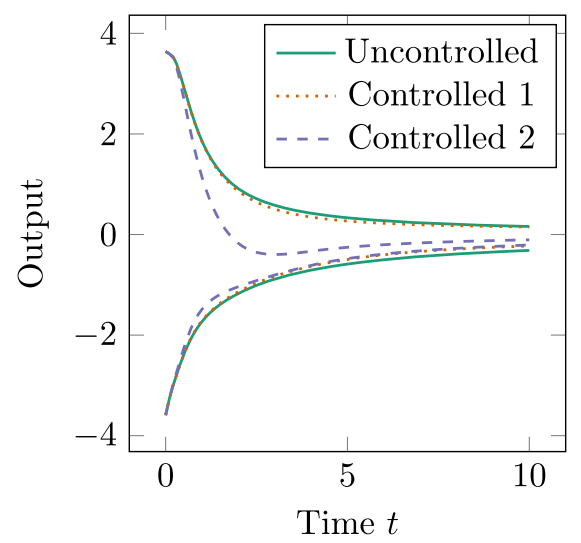

(a) Outputs for the same initial configuration for two different parameters.

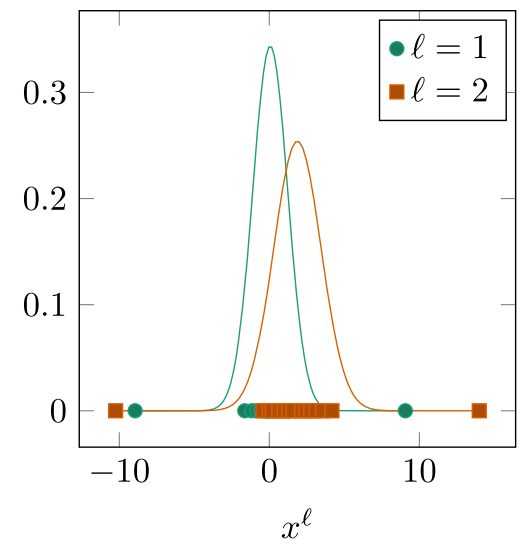

(b) Grids and distributions for the reduced domain.

Fig. 9 Test 3: Outputs of the controlled and uncontrolled example and the distributions and grids that were generated

step $\Delta t=5 \cdot 10^{-3}$. For this example, we again have to specify a distribution of initial values of interest. In both components, we pick Gaussian distributions with a covariance matrix given by equation (18) with $c=0.2, b=1$ and $\gamma=1$. In the second component we furthermore set the mean of the distribution to -1 yielding flows which are mostly directed from top to bottom. Figure 8 shows an example simulation, both the uncontrolled and HJB-controlled case. In Fig. 9a, we show two outputs for two different parameter configuration.

The ROM is built upon the LRFG algorithm on the linearized equation from (21). The algorithm yields a surrogate model of dimension $\ell=2$ and does not perform a parameter partitioning. We note that the linearization of (21) around the origin leads to the heat equation which is possible to reduce with a few basis functions. For the numerical domain of the reduced HJB equation, we compute 100 uncontrolled test simulations where the initial values were chosen according to the distribution $\mathcal{D}$. Then, in each dimension the reduced domain is discretized with 15 points according to the distributions that were estimated from the reduced coordinates of the test simulations (see Fig. 9b).

We build the feedback control from the value function that was obtained from our procedure and run test examples. Table 2 shows the improvement of our method by

Table 2 Test 3: The mean, minimum and maximum ratio $J^{\mathrm{C}} / J^{\mathrm{UC}}$ of the uncontrolled to the controlled cost for 10 randomly chosen initial values. Row-wise parameters: $\mu=(a, a)$ with $a=0.01,2.5,5$

\begin{tabular}{llll}
\hline & Mean & Best & Worst \\
\hline$a=0.1$ & 0.985 & 0.969 & 0.996 \\
$a=2.5$ & 0.720 & 0.512 & 0.903 \\
$a=5$ & 0.695 & 0.514 & 0.895 \\
\hline
\end{tabular}


Table 3 Test 3: The mean, minimum and maximum ratio $J^{\mathrm{LQR}} / J^{\mathrm{UC}}$ of the uncontrolled to the controlled cost for 10 randomly chosen initial values. Row-wise parameters: $\mu=(a, a)$ with $a=0.01,2.5,5$

\begin{tabular}{llll}
\hline & Mean & Best & Worst \\
\hline$a=0.1$ & 1.108 & 0.907 & 1.580 \\
$a=2.5$ & 0.997 & 0.779 & 1.612 \\
$a=5$ & 0.992 & 0.771 & 1.611 \\
\hline
\end{tabular}

evaluating the cost functional of the controlled problem over the cost functional of the uncontrolled problem. We ran the simulation with 10 different initial conditions for each parameter configuration and computed the mean (Column 1), the minimum (Column 2) and the maximum (Column 3) ratio $J^{\mathrm{C}} / J^{\mathrm{UC}}$ of the cost functional value for the uncontrolled dynamics $J^{\mathrm{UC}}$ and controlled dynamics $J^{\mathrm{C}}$. Furthermore, in Table 3, we provide the ratio $J^{\mathrm{LQR}} / J^{\mathrm{UC}}$ also for the LQR approach. We, clearly, see an improvement over the uncontrolled dynamics for increasing the weights in the cost functional when using the HJB approach. It is noteworthy that for almost all parameter configurations and initial values, the LQR controlled dynamics lead to larger cost functional values than the uncontrolled dynamics, which highlights the suboptimality of the LQR control for this scenario (see Table 2).

\subsection{About the calculation times}

Finally, we want to make general remarks about the CPU time, and in particular we discuss the benefit of the offline-online decomposition. We show in Table 4 those results. The second, third and fourth column refer to the offline costs to compute the basis functions, the VI in the barycenter of each subregion of the parameter space and to precompute the quantities of the affine decomposition, respectively. We then show the time needed to compute the value function with the PI algorithm. Furthermore, we show the benefit of the precalculation that speeds up the convergence of the method. All numbers present average measurements over 10 parameters drawn randomly from the parameter sets. We can observe a speed-up of factor 2 in the first case, of factor 13 in the second case and of 7 in the third case.

Table 4 Table for offline and online calculation times (in seconds) for all examples

\begin{tabular}{lllllll}
\hline & \multicolumn{3}{l}{ Offline Stage } & & & \multicolumn{2}{l}{ Online Stage } & \\
\cline { 2 - 3 } \cline { 6 - 7 } & Basis gen. & VI & Precalc. & & PI (no precalc.) & PI (precalc.) \\
\hline Test 1 & 480 & 72 & 99 & & 21 & 11 \\
Test 2 & 12 & 160 & 7 & & 161 & 13 \\
Test 3 & 52 & 25 & 0.3 & & 4.5 & 0.6 \\
\hline
\end{tabular}




\section{Concluding remarks}

In this paper, we have presented a workflow for the computation of HJB based feedback control for parametrized PDEs. Feedback control via DPP suffers from the curse of dimensionality and, therefore, we make use of model reduction techniques. In the current work, we show that snapshots generated from low-rank-factors of solutions of Algebraic Riccati Equations allow to build basis functions that approximate the low-dimensional value function globally with respect to the parameter thanks to the greedy parameter sampling. This approach turns out to be very general for the computation of the control for arbitrary initial conditions. We have also presented an automatic way to generate the reduced domain for the HJB equation.

As perspective we see a possible (probabilistic) analysis of the accuracy of the procedure for determining the reduced domains. To improve the offline computational times, predictive adaptivity control may be applied for the parameter domain partitioning, i.e., suitable early-stopping greedy procedures based on error decay monitoring. Another possible improvement will be the use of recent techniques based on a mesh-free approach [35] that allows to deal with problems in dimension larger than $4-5$ as we have in this paper.

Acknowledgments The authors would like to thank Max Gunzburger for several fruitful discussions on the subject and valuable comments for improvement of the presentation.

Funding information The first author was supported by US Department of Energy grant number DESC0009324. The second and third authors would like to thank the German Research Foundation (DFG) for financial support of the project within the Cluster of Excellence in Simulation Technology (EXC 310/2) at the University of Stuttgart.

\section{References}

1. Alla, A., Falcone, M., Kalise, D.: An efficient policy iteration algorithm for dynamic programming equations. SIAM J. Sci. Comput. 37, A181-A200 (2015). https://doi.org/10.1137/130932284

2. Alla, A., Falcone, M., Kalise, D.: A HJB-POD feedback synthesis approach for the wave equation. Bulletin of the Brazilian Mathematical Society New Series 47, 51-64 (2016). https://doi.org/10.1007/ s00574-016-0121-6

3. Alla, A., Falcone, M., Saluzzi, L.: An efficient DP algorithm on a tree-structure for finite horizon optimal control problems. SIAM J. Sci. Comput. 41, A2384-A2406 (2019). https://doi.org/10.1137/18M1203900

4. Alla, A., Falcone, M., Volkwein, S.: Error analysis for POD approximations of infinite horizon problems via the dynamic programming approach. SIAM J. Control. Optim. 55, 3091-3115 (2017). https://doi.org/10.1137/15M1039596

5. Alla, A., Schmidt, A., Haasdonk, B.: Model order reduction approaches for infinite horizon optimal control problems via the HJB equation. In: Benner, P., Ohlberger, M., Patera, A., Rozza, G., Urban, K. (eds.) Model Reduction of Parametrized Systems, pp. 333-347. Springer International Publishing, Cham (2017). https://doi.org/10.1007/978-3-319-58786-8_21

6. Antoulas, A.: Approximation of large-scale dynamical systems. SIAM Publications, Philadelphia (2005). https://doi.org/10.1137/1.9780898718713

7. Atwell, J., King, B.: Proper orthogonal decomposition for reduced basis feedback controllers for parabolic equations. Math. Comput. Model. 33, 1-19 (2001). https://doi.org/10.1016/s0895-7177(00) 00225-9 
8. Bardi, M., Capuzzo-Dolcetta, I.: Optimal control and viscosity solutions of Hamilton-Jacobi-Bellman Equations. Birkhäuser, Boston (1997). https://doi.org/10.1007/978-0-8176-4755-1

9. Barrault, M., Maday, Y., Nguyen, N., Patera, A.: An "empirical interpolation" method: application to efficient reduced-basis discretization of partial differential equations. Comptes Rendus de l'Académie des Sciences Series I 339, 667-672 (2004). https://doi.org/10.1016/j.crma.2004.08.006

10. Bellman, R. Dynamic Programming, 1st edn. Princeton University Press, Princeton (1957)

11. Benner, P., Gugercin, S., Willcox, K.: A survey of projection-based model reduction methods for parametric dynamical systems. SIAM Rev. 57, 483-531 (2015). https://doi.org/10.1137/130932715

12. Benner, P., Heiland, J.: LQG-balanced truncation low-order controller for stabilization of laminar flows. In: King, R. (ed.) Active Flow and Combustion Control 2014, vol. 127, pp. 365-379. Springer International Publishing (2015)

13. Bokanowski, O., Maroso, S., Zidani, H.: Some convergence results for Howard's algorithm. SIAM J. Numer. Anal. 47, 3001-3026 (2009). https://doi.org/10.1137/08073041X

14. Breiten, T., Kunisch, K.: Riccati-based feedback control of the monodomain equations with the Fitzhugh-Nagumo model. SIAM J. Control. Optim. 52, 4057-4081 (2014). https://doi.org/10.1137/ 140964552

15. Dedé, L.: Reduced basis method and a posteriori error estimation for parametrized linear-quadratic optimal control problems. SIAM J. Sci Comput. 32, 997-1019 (2010). https://doi.org/10.1137/09076 0453

16. Eftang, J.L., Patera, A.T., Rønquist, E.M.: An $h p$ certified reduced basis method for parametrized elliptic partial differential equations. SIAM J. Sci. Comput. 32, 3170-3200 (2010). https://doi.org/10.1137/090780122

17. Falcone, M.: A numerical approach to the infinite horizon problem of deterministic control theory. Appl. Math. Optim. 15, 1-13 (1987). https://doi.org/10.1007/BF01442644

18. Falcone, M., Ferretti, R.: Semi-lagrangian approximation schemes for linear and Hamilton-Jacobi equations, Society for industrial and applied mathematics. https://doi.org/10.1137/1.9781611973051 (2013)

19. Garcke, J., Kröner, A.: Suboptimal feedback control of PDEs by solving HJB equations on adaptive sparse grids. J. Sci. Comput. 70, 1-28 (2017). https://doi.org/10.1007/s10915-016-0240-7

20. Grüne, L., Pannek, J.: Nonlinear model predictive control. In: Nonlinear Model Predictive Control, pp. 43-66. Springer, London (2011). https://doi.org/10.1007/978-0-85729-501-9_3

21. Gubisch, M., Volkwein, S.: POD for linear-quadratic optimal control. In: Benner, P., Cohen, A., Ohlberger, M., Willcox, K. (eds.) Model Reduction and Approximation: Theory and Algorithms. SIAM, Philadelphia (2017)

22. Haasdonk, B.: Convergence rates of the POD-Greedy method. ESAIM: Mathematical modelling and numerical Analysis 47, 859-873 (2013). https://doi.org/10.1051/m2an/2012045

23. Haasdonk, B., Dihlmann, M., Ohlberger, M.: A training set and multiple basis generation approach for parametrized model reduction based on adaptive grids in parameter space. Math. Comput. Model. Dyn. Syst. 17, 423-442 (2011). https://doi.org/10.1080/13873954.2011.547674

24. Hinze, M., Pinnau, R., Ulbrich, M., Ulbrich, S.: Optimization with PDE Constraints. Mathematical Modelling Theory and Applications. Springer, Berlin (2009). https://doi.org/10.1007/978-1-40208839-1

25. Howard, R.A.: Dynamic Programming and Markov processes. MIT Press, Cambridge (1960)

26. Junge, O., Schreiber, A.: Dynamic programming using radial basis functions. Discrete and Continuous Dynamical Systems 35, 4439-4453 (2015). https://doi.org/10.3934/dcds.2015.35.4439

27. Kalise, D., Kröner, A.: Reduced-order minimum time control of advection-reaction-diffusion systems via dynamic programming. In: 21st International Symposium on Mathematical Theory of Networks and Systems, Groningen, Netherlands. https://hal.archives-ouvertes.fr/hal-01089887, pp. 1196-1202 (2014)

28. Kalise, D., Kunisch, K.: Polynomial approximation of high-dimensional Hamilton-Jacobi-Bellman equations and applications to feedback control of semilinear parabolic PDEs. SIAM J. Sci. Comput. 40, A629-A652 (2018). https://doi.org/10.1137/17M1116635

29. Kärcher, M., Grepl, M.: A posteriori error estimation for reduced order solutions of parametrized parabolic optimal control problems. ESAIM: Mathematical Modelling and Numerical Analysis 48, 1615-1638 (2014). https://doi.org/10.1051/m2an/2014012 
30. Kunisch, K., Volkwein, S., Xie, L.: HJB-POD based feedback design for the optimal control of evolution problems. SIAM Journal on Applied Dynamical Systems 3, 701-722 (2004). http://epubs.siam. org/doi/abs/10.1137/030600485

31. Kunisch, K., Xie, L.: POD-based feedback control of the Burgers equation by solving the evolutionary HJB equation. Computers \& Mathematics with Applications 49, 1113-1126 (2005). https://doi.org/10.1016/j.camwa.2004.07.022

32. Patera, A., Rozza, G.: Reduced basis approximation and a Posteriori Error Estimation for Parametrized Partial Differential Equations, To appear in (tentative) MIT Pappalardo Graduate Monographs in Mechanical Engineering, MIT. http://augustine.mit.edu/methodology/methodology_book.htm (2007)

33. Ravindran, S.S.: A reduced order approach for optimal control of fluids using proper orthogonal decomposition. Int. J. Numer. Methods Fluids 34, 435-448 (2000). https://doi.org/10.1002/1097-0363 (20001115)34:5<425::AID-FLD67>3.0.CO;2-W

34. Santos, M.S., Rust, J.: Convergence properties of policy iteration. SIAM J. Control. Optim. 42, 2094 2115 (2004). https://doi.org/10.1137/S0363012902399824

35. Schmidt, A., Haasdonk, B.: Data-driven surrogates of value functions and applications to feedback control for dynamical systems. IFAC-PapersOnLine 51, 307-312 (2018). https://doi.org/10.1016/j. ifacol.2018.03.053

36. Schmidt, A., Haasdonk, B.: Reduced basis approximation of large scale parametric algebraic Riccati equations. ESAIM: Control Optimisation and Calculus of Variations 24, 129-151 (2018). https://doi.org/10.1051/cocv/2017011

37. Simoncini, V.: Analysis of the rational Krylov subspace projection method for large-scale algebraic Riccati equations. SIAM Journal on Matrix Analysis and Applications 37, 1655-1674 (2016). https://doi.org/10.1137/16m1059382

38. Tröltzsch, F.: Optimal Control of Partial Differential Equations. Theory, Methods and Applications, vol. 112 of Mathematical Modelling: Theory and Applications. American Mathematical Society, Providence (2010)

39. Tröltzsch, F., Volkwein, S.: POD a-posteriori error estimates for linear-quadratic optimal control problems. Comput. Optim. Appl. 44, 83-115 (2009). https://doi.org/10.1007/s10589-008-9224-3

Publisher's note Springer Nature remains neutral with regard to jurisdictional claims in published maps and institutional affiliations.

\section{Affiliations}

\section{Alessandro Alla ${ }^{1}$ D $\cdot$ Bernard Haasdonk ${ }^{2}$. Andreas Schmidt ${ }^{2}$}

Bernard Haasdonk

haasdonk@mathematik.uni-stuttgart.de

Andreas Schmidt

schmidta@mathematik.uni-stuttgart.de

1 Department of Mathematics, PUC-Rio, Rua Marques de São Vicente, 225 Rio de Janeiro, 22453-900, Brazil

2 Institute of Applied Analysis and Numerical Simulation, University of Stuttgart, Pfaffenwaldring 57, 70569, Stuttgart, Germany 